was to improve the quality of our transition service by increasing knowledge of the transition process and surpassing the NICE Quality Standard (QS140).

Methods 3 evening events have been held. We have invited 24 young people and their carers to each event. Event structure includes height and weight measurement, lung function and/or inhaler technique and appointment with Respiratory Consultant or Specialist Nurse.

Young people were seen alone as part of the consultation. They were given information, introduced to the Ready Steady Go process and questionnaires were completed. Outcome measures were based on attendance rates, questionnaire feedback and achievement of the NICE Quality Standard.

Results Attendance has been variable. Maximal rates were achieved when appointment letter and personal phone calls were used.

\begin{tabular}{lccc}
\hline Clinic & June 2017 & October 2017 & June 2018 \\
\hline Attendance Rate & $33 \%$ & $82.4 \%$ & $50 \%$ \\
\hline
\end{tabular}

The event was very popular with young people and their carers. After all 3 events, $100 \%$ of young people understood what transition is and our local approach. $100 \%$ of young people felt ready for transition. $100 \%$ carers understood the process of transition, felt their child is ready to build independence and found the event useful. The event provides an opportunity to deliver the NICE Quality Standard. For patients who attended, $100 \%$ have a transition plan (QS1401), 100\% have had an annual meeting (QS140-2) and 100\% have been introduced to our named worker (QS140-3).

Conclusion Our model is popular with young people and their carers. This is a useful way to achieve the Quality Standard for patients in attendance but is time-consuming and labour intensive. The model can be applied to other specialties and to multi-speciality events. We hope to introduce a worker from adult services at our future events to further improve achievement of the NICE Quality Standard QS140-4.

\section{P39 MEETING THE SEXUAL HEALTH NEEDS OF ADOLESCENTS}

${ }^{1} \mathrm{E}$ Barr*, ${ }^{1} \mathrm{M}$ Rooney, ${ }^{1} \mathrm{M}$ Moore, ${ }^{2} \mathrm{~K}$ Zipperer. ${ }^{1}$ Public Health, University of North Florida, Jacksonville, USA; ${ }^{2}$ Office of Healthy Schools, Florida Department of Education, Tallahassee, USA

\subsection{6/bmjpo-2019-RCPCH-SAHM.43}

Aims This study assessed support for increases in sexuality education and sexual health services for both middle school and high school students. Data is essential to reinforce change in communities with limited sexuality education, services, and related policy, yet with high rates of teen pregnancy and STDs/HIV.

Methods A 22-item survey was developed after an extensive review of existing instruments and feedback from an expert panel. Questions addressed support for specific sexuality education topics and sexual health services at middle and high school levels. Through a University research-polling center, Random-Digit-Dialing methodology for landlines (26.9\%) and cell phones $(71.3 \%)$ was implemented. Surveys were conducted in English and Spanish. The resulting sample included 615 completed surveys.

Results Despite wide differences in party identification, median household income, population density and racial make-up, the counties' aggregate opinions were very supportive of sexuality education and sexual health services. Of eight sexuality education topics presented, 'HIV and STDs' received the highest support (91\% middle school, 96\% high school) and 'Gender and Sexual Orientation' received the lowest $(71 \%$ middle school, 78\% high school). Of four sexual health services presented, 'Testing for STDs/HIV' received the highest level of support (61\% middle school, $82 \%$ high school), while 'Providing Condoms' received the lowest (49\% middle school, 69\% high school). Additionally, most participants (84-90\%) would allow their children to participate in grade level appropriate sexuality education, most (85-89\%) support teaching both abstinence and birth control, yet almost half (47\%) do not know if sexuality education is taught in their districts. Demographic differences will be presented along with participant perceptions of adolescent sexual behaviors.

Conclusions Overwhelming support for increasing efforts to improve sexual health among adolescents exits. Findings from this study can be used to advance initiatives addressing unplanned pregnancy, STD/HIV infection, healthy relationships and active consent. This data supports components of evidence-based programs and current national efforts to improve adolescent sexual heath.

\section{P40 IMPACT OF A BRIEF VIDEO TO IMPROVE NALOXONE KNOWLEDGE IN ADOLESCENTS AND YOUNG ADULTS WITH SEVERE OPIOID USE DISORDER}

${ }^{1,2}$ E McKnight* ${ }^{*}{ }^{1,2,3} \mathrm{~A}$ Bonny, ${ }^{1,2} \mathrm{~S}$ Matson, ${ }^{1,2} \mathrm{C}$ Cottrill. ${ }^{1}$ Adolescent Medicine, Nationwide Children's Hospital, Columbus, USA; ${ }^{2}$ Pediatrics, The Ohio State University, Columbus, USA;

${ }^{3}$ The Research Institute, Nationwide Children's Hospital, Columbus, USA

\subsection{6/bmjpo-2019-RCPCH-SAHM.44}

Aims Humanity has experienced several opioid crises, but none as devastating as the present one mainly affecting North America. Opioid overdose death rates among United States' youth have tripled since 1999. The aim of this study was to assess the impact of a short educational video on knowledge of naloxone for opioid overdose resuscitation among adolescents and young adults (AYA) with severe opioid use disorder.

Methods AYA receiving outpatient, medication-assisted treatment for severe opioid use disorder were provided a brief educational video when prescribed a naloxone overdose treatment kit. The video reviewed the correct signs of overdose, overdose risks, proper use of naloxone, and order of resuscitation steps. A 5-item survey assessed naloxone knowledge preand post-video. A self-completed survey obtained information regarding drug use and overdose experiences. McNemar's test was used to compare the proportion with correct answers pre- and post-video.

Results Of 35 participating AYA, 26 (74\%) were female and 32 (91\%) white, non-Hispanic. Twenty-two (65\%) had witnessed a drug overdose. Of witnessed resuscitation efforts reported, 15 (43\%) observed Emergency Medical Service assistance, 12 (34\%) observed 'other efforts', and only 4 (11\%) reported witnessing naloxone administration. Five (14\%) AYA had experienced a personal drug overdose; the mean number of overdoses was $4( \pm 3.32)$ with heroin being the most 